\title{
Comprehensive critical thinking problem solving assessment framework for engineering students
}

\begin{abstract}
This paper proposed a framework to assess Critical Thinking Problem Solving (CTPS) among Computer and Communication Systems Engineering students. Currently, CTPS is assessed using generic rubric that can be interpreted differently. The proposed framework consists of in-class oral, facilitation and report assessment methods that tackle the limitation of current assessment practice. After three assessments are performed throughout the semester, the total score is the accumulation of the three assessment scores. The study was implemented at Faculty of Engineering, UPM in Semester 12016/2017 which involves 41 undergraduate students, ranging from second to final years students in 7 high taxonomy level courses. From the conducted study, the proposed CTPS assessment method is comprehensive covering the oral, facilitation and report aspect. However, the proposed framework is difficult to be conducted for large classroom due to the time constraint but the data obtained in the study can be used to propose an efficient and reliable rubric for CTPS assessment.
\end{abstract}

Keyword: Critical thinking skills; Oral assessment; Facilitation assessment; Report assessment 